\title{
Mejora docente e incremento de la visibilidad en la ingeniería naval e industrial a través del uso de instalaciones experimentales
}

\author{
Míguez González, Marcos; Gosset, Anne; Díaz Casás, Vicente; Lema Rodríguez, \\ Marcos; Santiago Caamaño, Lucía \\ Universidade da Coruña, Escola Politécnica Superior
}

\section{RESUMEN}

El Grupo de Innovación Educativa CITEX de la Universidade da Coruña está formado por docentes de las titulaciones relacionadas con la Ingeniería Naval y Oceánica y la Ingeniería Industrial que se imparten en la Escola Politécnica Superior de Ferrol.

Sus objetivos principales consisten en mejorar los resultados académicos y la visibilidad y atractivo de estas titulaciones, que tradicionalmente no han sido elevados, mediante la utilización de nuevos recursos metodológicos que mejoren y actualicen las metodologías de enseñanza utilizadas, entre los que se encuentran el uso en las actividades de enseñanza de las instalaciones experimentales singulares de investigación. Estas instalaciones incluyen el canal de ensayos hidrodinámicos, los dos túneles de viento y una instalación de análisis de cavitación en válvulas situados en el Centro de Investigaciones Tecnológicas (CIT) de la Universidade da Coruña, en Ferrol.

En este trabajo, se describirán las actividades de enseñanza realizadas dentro de varias asignaturas de estas titulaciones para incrementar las tasas de éxito de las mismas, así como las actividades de difusión y transferencia, dirigidas a un amplio espectro de público objetivo, que buscan mejorar la percepción que de dichas titulaciones y de la ciencia tienen su potencial alumnado.

PALABRAS CLAVE: divulgación científica; difusión de titulaciones; recursos experimentales. 


\section{CITA RECOMENDADA:}

Míguez González, Marcos; Gosset, Anne; Díaz Casás, Vicente; Lema Rodríguez, Marcos; Santiago Caamaño, Lucía (2020): Mejora docente e incremento de la visibilidad en la ingeniería naval e industrial a través del uso de instalaciones experimentales. En De la Torre Fernández, E. (ed.) (2020). Contextos universitarios transformadores: Boas prácticas no marco dos GID. IV Xornadas de Innovación Docente. Cufie. Universidade da Coruña. A Coruña (págs. 405-420).

DOI capítulo: https://doi.org/10.17979/spudc. 9788497497756.405

DOl libro: https://doi.org/10.17979/spudc. 9788497497756

\section{ABSTRACT}

The Education Innovation Group CITEX of the University of A Coruña is composed of professors and research assistants from degrees related to Marine Engineering and Naval Architecture and Industrial Engineering, which are taught in the Higher Polytechnic School in Ferrol.

The main objectives of this group include improving the academic results of the students of these degrees and their visibility and attractiveness, which have been historically low. These goals are proposed to be obtained by using singular experimental research facilities: a hydrodynamic towing tank, two wind tunnels and a valve cavitation system, all placed in the Centre for Technological Research (CIT) of the University of A Coruña in Ferrol.

In this work, the activities done within some courses of these degrees for increasing their success rates, together with the dissemination of these degrees and the research facilities directed to a broad spectrum of public, are described.

KEY WORDS: scientific dissemination; degrees dissemination; experimental resources. 


\section{INTRODUCCIÓN}

Las titulaciones del campo de la Ingeniería Naval y de la Ingeniería Industrial que se imparten en la Escuela Politécnica Superior de la Universidad de la Coruña, sita en el Campus de Esteiro en Ferrol, vienen a presentar, históricamente, unas bajas tasas de éxito en muchas de sus materias y problemas en los niveles de matrícula, debido tanto a la situación geográfica del centro, como al desconocimiento de las titulaciones que se imparten en el mismo y sus posibilidades laborales, lo cual se enmarca en un contexto más general que es la drástica disminución en el número de matrículas universitarias en grados de Ciencia, Tecnología, Ingeniería y Matemáticas (S.T.E.M.) (OECD, 2008). Más específicamente, en España, en el curso académico 2014-2015, se ha registrado una disminución del 28,7\% y un 22,8\% de matrículas en estudios de ingeniería y ciencias con respecto al curso 2004-2005, respectivamente (MECD, 2016).

Con el objetivo de mejorar la calidad de la enseñanza y de renovar el modelo educativo utilizado en sus titulaciones, la Universidade da Coruña publicó en 2017 la primera convocatoria de Grupos de Innovación Docente (GIDs) y que tuvo su continuación en 2018. Dentro de esta segunda convocatoria, se enmarca el GID CITEX.

Este Grupo de Innovación Docente está formado por docentes y colaboradores de las titulaciones de grado y master relacionadas con la Ingeniería Naval y Oceánica y la Ingeniería Industrial, y todos ellos centran parte de su actividad investigadora en una serie de instalaciones experimentales singulares que se disponen en el Centro de Investigaciones Tecnológicas de la UDC (CIT) del Campus de Esteiro: el Canal de Ensayos Hidrodinámicos y el laboratorio de Mecánica de Fluidos, que incluye dos túneles de viento y un sistema de observación de cavitación en sistemas de tuberías. Este Grupo de Innovación Docente se formó con tres objetivos principales. En primer lugar, la mejora de los resultados académicos del alumnado de estas titulaciones, en lo que se refiere a las materias relacionadas con la mecánica de fluidos (campo industrial) y la hidrostática y la estabilidad del buque (campo naval). En segundo lugar, a contribuir a la difusión y conocimiento de estos títulos no sólo en 
la ciudad de Ferrol, sino también a nivel provincial e incluso autonómico y nacional. Y, por último, a divulgar y transferir al entorno del centro las tareas de investigación que en él se llevan habitualmente a cabo.

En relación a la obtención de avances en la metodología docente, las tareas realizadas incluían el diseño de una serie de actividades de enseñanza adicionales a las llevadas a cabo en el aula que, mediante el uso de estas instalaciones, proporcionasen al alumnado no sólo una mejor comprensión de algunos conceptos teóricos de gran importancia de las diferentes materias, sino también un conocimiento básico de la investigación aplicada que se lleva a cabo en la Universidad y de cómo esta puede contribuir el avance de la técnica activando un modelo de enseñanza teórico-práctico que favorezca el necesario cambio conceptual y actitudinal y el aprendizaje significativo (Caamaño, 2012).

En lo que se refiere a las actividades de divulgación, el incremento del conocimiento de las titulaciones y la transferencia de conocimiento, las actividades planteadas incluían la realización de ensayos y exhibiciones sencillas desde el punto de vista técnico pero de gran contenido e impacto visual, habida cuenta del heterogéneo público objetivo al que se dirigían: estudiantes de educación primaria, de educación secundaria y bachillerato, padres y familiares de los anteriores y público en general, no interesado en el acceso a las titulaciones pero sí en las actividades que se realizan en la Universidad.

En ambos casos, las actividades que se presentan se realizaron durante el curso 2018/2019 (septiembre de 2018 a septiembre de 2019). Respecto a los resultados referidos a los avances en las tasas de éxito de las distintas materias, a pesar de que la información subjetiva obtenida del alumnado fue muy positiva, la influencia en las tasas de éxito de las distintas materias puede sólo considerarse cómo moderadamente positiva. Por otra parte, las tareas de divulgación desarrolladas sí que obtuvieron unos resultados muy satisfactorios, incrementando en la comarca y autonomía la visibilidad de las actividades realizadas en el Campus, así como contribuyendo a la matrícula y atracción de alumnos a las titulaciones de los ámbitos mencionados. 


\section{ACTIVIDADES DE MEJORA DE CALIDAD DE LA DOCENCIA}

Dentro de las tareas relacionadas con el incremento de la calidad de la docencia, estas se pueden dividir en dos grupos diferentes. Unas relacionadas con las titulaciones de grado y máster en Ingeniería Naval y Oceánica, y que se realizan utilizando el Canal de Ensayos Hidrodinámicos del CIT de Ferrol. Y un segundo grupo enfocado a las titulaciones de grado y máster del ámbito de la ingeniería industrial (grado en Ingeniería Mecánica, grado en Ingeniería en Tecnologías Industriales y master en Ingeniería Industrial), y que se realizan utilizando el laboratorio de fluidos del CIT (Túnel de Viento e instalaciones experimentales de visualización de cavitación). Las materias y titulaciones en que se han aplicado estas acciones se enumeran en la Tabla 1.

\begin{tabular}{c|c|c}
\hline Materia & Curso & Titulación \\
\hline Hidrostática y Estabilidad & $2^{0}$ & Grado en Ingeniería Naval y Oceánica \\
Ampliación de Hidrostática e & $1^{0}$ & Máster en Ingeniería Naval y Oceánica \\
Hidrodinámica & $1^{0}$ & Máster en Ingeniería Naval y Oceánica \\
Estabilidad tras Averías & $2^{0}$ & Grado en Ingeniería Mecánica / Grado en Ing. en \\
Mecánica de Fluidos & $3^{0}$ & tecnologías Industriales \\
Máquinas Térmicas e Hidráulicas & & tecnologías Industriales \\
\hline
\end{tabular}

Tabla 1. Materias en que se realizaron actividades prácticas utilizando las instalaciones singulares de investigación.

El objetivo de las actividades planteadas buscaba no sólo dar cumplimiento a uno de los pilares de Delors (Delors, 1996) en los que se debe basar la educación, Aprender a conocer 0 saber (no acumular conocimientos sino dominar los instrumentos del saber), sino también asentar algunos de los principios básicos del curriculum del estudiante según el Espacio Europeo de Educación Superior (EEES), que establece que debe ser competitivo, incorporando la evaluación, la investigación y la innovación como recursos para "[...] estimular la excelencia en general y permitir a las universidades que desempeñen su papel como socios esenciales en pro del desarrollo social, económico y cultural" (Conference of Ministers responsible for Higher Education, 2005), y centrado en el alumno, reconociendo sus conocimientos previos, 
considerando cuando sea posible el aprendizaje formal o no-formal (Universidade da Coruña, 2013) e introduciendo métodos de enseñanza innovadores (European University Association, 2003.).

Las actividades experimentales se plantearon para facilitar la asimilación de una serie de elementos problemáticos y de especial relevancia de entre los contenidos de cada una de las materias, que en algunos casos podrían llegar a considerarse como "Threshold Concepts" (Knight et al, 2014; Land, Meyer \& Flanagan, 2016), y que son fundamentales no sólo para la obtención de las competencias especificadas en cada asignatura, si no para la comprensión de gran parte de los contenidos de las mismas.

En el caso de las materias relacionadas con la Ingeniería Naval y Oceánica, en la asignatura de Hidrostática y Estabilidad se imparten los fundamentos de flotabilidad, estabilidad y seguridad del buque, que serán de aplicación directa en la vida laboral de los egresados y serán también necesarios en materias que continúan el curriculum en cursos superiores. La materia de Ampliación de Hidrostática e Hidrodinámica es una materia de complementos de formación para alumnado que accede al master desde titulaciones de grado en donde no se han adquirido todos los contenidos necesarios de Hidrostática y Estabilidad y de Hidrodinámica. Teniendo en cuenta que las actividades planteadas están relacionadas principalmente con la Hidrostática y Estabilidad, son comunes a las dos materias mencionadas. En estas materias, los nuevos recursos metodológicos consistieron en actividades de enseñanza diseñadas para comprobar mediante la experimentación con modelos demostradores a escala los conceptos teóricos que se estudian teóricamente en las clases magistrales, intercalando visitas al Canal de Ensayos con clases teóricas. Los elementos de especial relevancia que se estudian con estas actividades son las curvas de estabilidad en estado intacto del buque (GZ), la estabilidad dinámica, el efecto que tienen tanto la distribución de pesos como las superficies libres sobre la estabilidad del buque (Biran and López-Pulido, 2014). En la tercera de las materias relacionadas con la Ingeniería Naval y Oceánica (Estabilidad tras Averías, master en Ingeniería Naval y Oceánica), las actividades de enseñanza consistieron en la realización de ensayos con 
un modelo de buque a escala bajo la acción de olas en el Canal de Ensayos Hidrodinámicos, de modo que permitiese al alumnado no sólo observar, sino excitar modificando las características del buque y/o las olas, comportamientos dinámicos debidos a la interacción buque / olas que, salvo que se disponga de una instalación de este tipo, no pueden observarse en entornos controlados en ningún ámbito (Míguez and Bulian, 2017).

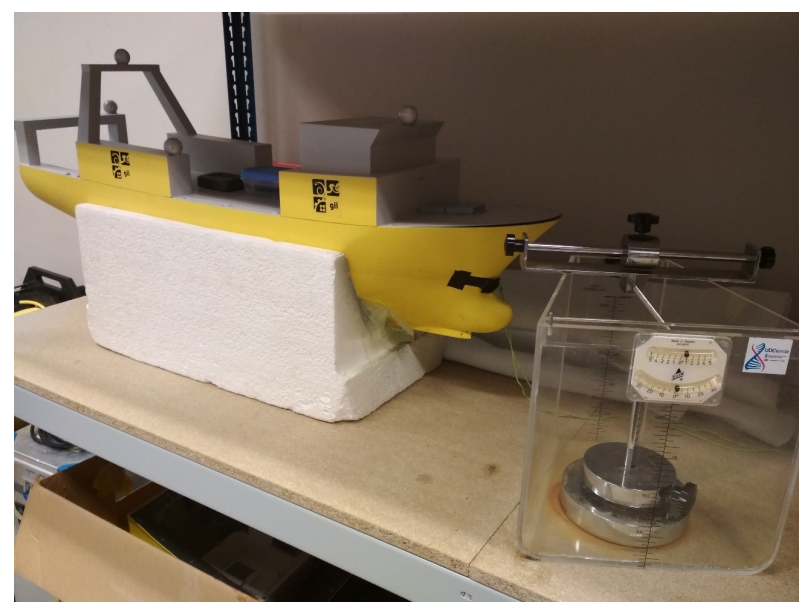

Figura 1. Modelo a escala de buque (izqda.) y demostrador (dcha.) utilizados para la realización de actividades formativas en el campo de la Ingeniería Naval y Oceánica.
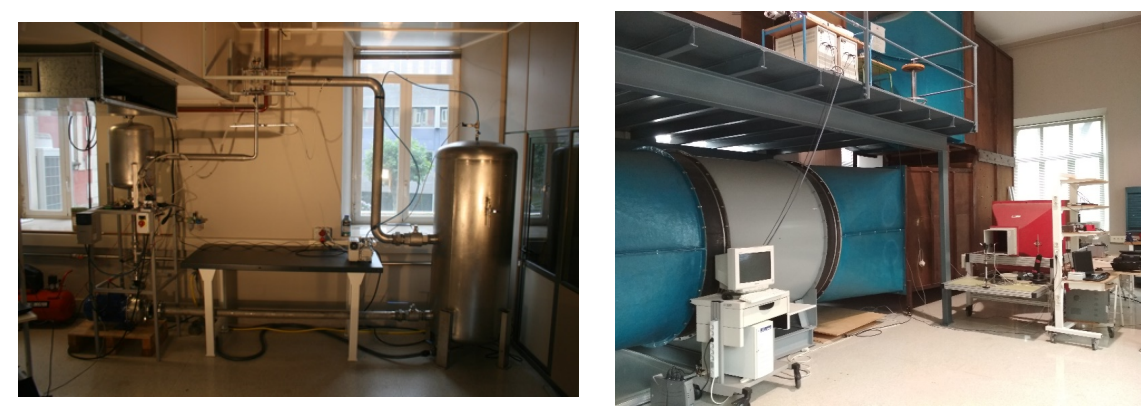

Figura 2. Instalaciones utilizadas para actividades formativas en el campo de la Ingeniería Industrial. Izqda.: instalación experimental de bombas y valvulería. Dcha.: túneles de viento.

Dentro del segundo grupo de asignaturas (relacionadas con la Ingeniería Industrial), las actividades de enseñanza realizadas se enmarcan en las materias de Mecánica de Fluidos y 
Máquinas Térmicas e Hidráulicas, que se imparten en los grados en Enxeñería Mecánica y Enxeñería en Tecnologías Industriales. Estas actividades consistieron en mostrar de forma práctica aspectos clave explicados de forma teórica en el aula, tales como la formación de capa límite, modelo a escala y análisis adimensional y, por último, pérdidas de carga en instalación hidráulicas. En concreto, la instalación experimental de bombas y valvulería, la cual está completamente sensorizada, permite los estudiantes comparar resultados teóricos obtenidos en el aula con las medidas realizadas en el laboratorio. Respecto a los resultados conseguidos por los nuevos recursos metodológicos propuestos, debe mencionarse que en el momento actual no se disponen de datos objetivos que permitan evaluar el grado de satisfacción por parte del alumnado con las actividades realizadas ni su impacto en la evaluación de las diferentes materias. Toda vez que este fue el primer curso de implantación del grupo de innovación docente, el trabajo en este punto se dirigió principalmente al diseño y puesta en marcha de las actividades experimentales, y no se elaboraron encuestas específicas dirigidas a ponderar de modo objetivo estos parámetros. Respecto a las encuestas de evaluación docente que la Universidade da Coruña difunde entre el profesorado y alumnado al final de cada cuatrimestre (programa UDC Avaliemosi), a pesar de que los resultados recibidos por los distintos docentes han sido positivos, en todos los casos por encima de la media de Universidad, Centro y Departamento, es importante destacar que el número de respuestas recibidas no ha superado, en ningún caso, el 10\% de los alumnos matriculados. Es por ello que estas encuestas, bajo estas condiciones, no pueden considerarse representativas. En todo caso, subjetivamente, las opiniones obtenidas de los estudiantes en el aula y tras la evaluación vienen siendo muy positivas respecto de estas actividades. Asimismo, y respecto a los resultados académicos, cabe destacar que los ratios obtenidos en las distintas materias no permiten concluir que las actividades realizadas hayan influido de modo claro en los mismos. Así, en la mayor parte de materias, las tasas de éxito o de no presentados se han mantenido en valores semejantes a los del curso anterior, por lo que tampoco se puede extraer de los mismos ningún efecto de las actividades realizadas. 
Estos hechos hacen pues imprescindible que, con el fin de disponer de una herramienta objetiva de evaluación de efectividad de las actividades planteadas, se plantee un programa de encuestas personalizadas que permitan obtener, de modo detallado, la influencia y el nivel de satisfacción que presentan los estudiantes respeto las actividades de enseñanza experimentales realizadas, así como pruebas objetivas u otras herramientas de evaluación que proporcionen capacidad de análisis sobre el grado de adquisición y manejo de los conceptos que se pretende reforzar con estas actividades.

\section{ACTIVIDADES DE DIVULGACIÓN Y DIFUSIÓN}

EI GID CITEX tenía, dentro de sus objetivos, la utilización de las instalaciones experimentales ya mencionadas para fines docentes, tareas que fueron descritas en el apartado anterior. Sin embargo, un segundo objetivo incluía la realización de tareas de promoción y difusión de las titulaciones de grado en que sus miembros están involucrados, así como de divulgación a la sociedad del conocimiento y de la investigación que se realiza en el Campus de Esteiro, en Ferrol, en sus respectivas especialidades (y especialmente en los laboratorios adscritos al CIT), con la finalidad de mejorar la percepción que la sociedad tiene de las carreras de ciencias para fomentar las vocaciones científicas entre los estudiantes de secundaria ya que se ha observado que en las últimas décadas, se viene registrado un generalizado desarrollo de actitudes negativas hacia la ciencia (Osborne, Simon y Collins, 2003).

Respecto a la difusión de las titulaciones de grado que se imparten en la Escola Politécnica Superior, cabe mencionar que en los últimos años (2014 - 2018), la matrícula en las mismas, salvo en el caso del grado en Ingeniería Mecánica en que se ha mantenido relativamente constante, ha experimentado un moderado descenso, acusado en el caso del grado en Ingeniería en Tecnologías Industriales, tal y como puede observarse en la Figura 4. Asimismo, y como puede observarse también en dicha figura, es también relevante el hecho de que en todas ellas el número de mujeres matriculadas haya disminuido continuamente. Esto puede ser debido, entre otras razones, a la situación geográfica de la Escola Politécnica Superior, situada en Ferrol y alejada de la sede principal de la UDC en A Coruña, a tratarse de 
titulaciones con tradición de dificultad y complejidad y mayoritariamente masculinas, al desconocimiento de los contenidos que se imparten en las mismas y su proyección e inserción laboral y, evidentemente, a la gran oferta de títulos disponibles en el sistema universitario autonómico y nacional, entre los que en muchas ocasiones es compleja la diferenciación. Al mismo tiempo, las instalaciones experimentales situadas en el CIT del Campus de Esteiro en Ferrol han pasado, históricamente, desapercibidas por la sociedad de la propia ciudad que las acoge. La necesidad de visibilizar la investigación a través de la divulgación no sólo es uno de los objetivos de las Universidades, sino que la comprensión pública de la ciencia es uno de los valores intrínsecos de las sociedades democráticas (Blanco López, 2004); asimismo, la mejora del conocimiento público de la ciencia derivará en una mayor aceptación de la misma y, por ende, en un mayor apoyo y subvención (Blanco López, 2004), lo cual es vital en estos últimos años y no ajeno a las instalaciones del CIT. Es por todo ello que, durante los años 2018 y 2019, los docentes adscritos al GID CITEX desarrollaron una serie de actividades, junto con los demás estamentos implicados (Escola Politécnica

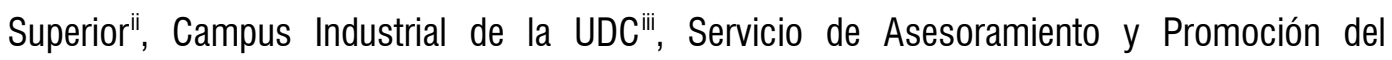
Estudiante de la UDC (SAPE) ${ }^{\text {iv }}$, Centro de Investigaciones Tecnológicas de la UDC (CIT) ${ }^{v}$, Xunta de Galicia) que incluían difusión y promoción de titulaciones así como divulgación científica, utilizando en las mismas siempre las instalaciones experimentales ya descritas en este trabajo. Por un lado, usando el Canal de Ensayos Hidrodinámicos, donde se dispuso un modelo a escala para ilustrar las actividades de ensayo que se realizan en la instalación y, al mismo tiempo, para difundir parte de los contenidos que se imparten en las titulaciones de grado y máster en Ingeniería Naval y Oceánica. Y por el otro, utilizando el Túnel de Viento, en el que con el uso de un anemómetro y un modelo a escala de un vehículo se ilustra tanto la aplicación de la instalación con tareas industriales e investigadoras, como posibles salidas laborales de los egresados de los grados y masters de la rama de la Ingeniería Industrial.

Estas jornadas y actividades incluyeron la participación en dos jornadas organizadas por el SAPE de Ferrol con el fin de dar a conocer el Campus de Esteiro, sus titulaciones y líneas de investigación, tres jornadas de puertas abiertas organizadas por la Escuela Politécnica 
Superior/Campus Industrial para dar a conocer las titulaciones y las actividades de la misma (Días de la Ingeniería), una jornada coincidente con la celebración de la First Lego League Galiciavi, en las que se mostraban a los niños participantes y sus familias los laboratorios e instalaciones a través de ensayos y experimentos prácticos, la participación en las campañas de inspiración de vocaciones científicas en las niñas, organizadas por la Xunta de Galicia y finalmente visitas concertadas específicamente con el CIT por parte de centros educativos tanto de la comarca como de fuera de la misma.

El análisis de la efectividad de estas actividades se puede agrupar habida cuenta la influencia en la matrícula de las titulaciones relacionadas con la Ingeniería Naval e Industrial que estas actividades buscaban promocionar, y considerando la difusión obtenida de las mismas hacia población general.

Respeto a este último punto, los resultados conseguidos son altamente satisfactorios, ya que estas actividades, y por lo tanto las titulaciones y tareas de investigación que en ellas se desarrollan, consiguieron una gran difusión en medios locales, autonómicos y nacionales. Dos ejemplos representativos pueden ser el haber sido portada del diario de gran tirada La Voz de Galicia en su edición de Ferrolvii 0 la aparición en los telediarios de la Televisión de Galicia viii. Respeto al efecto de estas acciones en la matrícula de estas titulaciones, pueden observarse los resultados que se presentan en la Figura 4, donde se puede apreciar la evolución histórica del número de alumnos matriculados en las distintas titulaciones objeto de promoción.
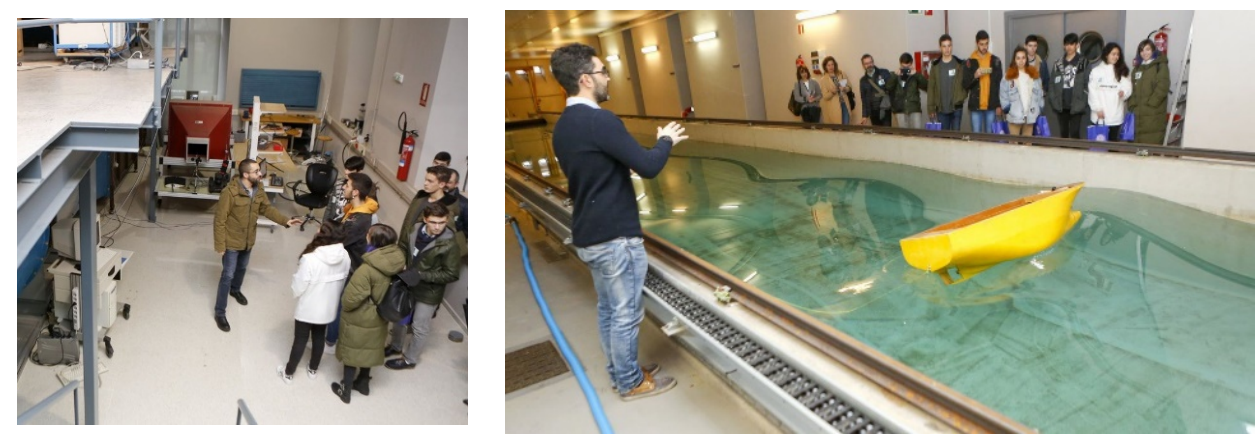

Figura 3. Tareas de divulgación en el Túnel de Viento (izquierda) y en el Canal de Ensayos Hidrodinámicos (derecha). Fuente: La Voz de Galicia vii. . 
Aunque este tipo de actividades se han venido realizando en menor medida en años anteriores, fue en 2018 y primera mitad de 2019 cuando se impulsaron definitivamente, y las anualidades 2018 y 2019 son las primeras en que podría apreciarse su efecto. Los datos mostrados en esta Figura 4 muestran un salto positivo en todas las titulaciones en la anualidad 2018, especialmente en comparación con los valores de descenso de matrícula observados hasta ese momento, mientras que en la anualidad 2019 se observa un nuevo descenso de matrícula, moderado en los grados de Ingeniería Naval y Oceánica y Tecnologías Industriales, y más acusado en Ingeniería Mecánica.
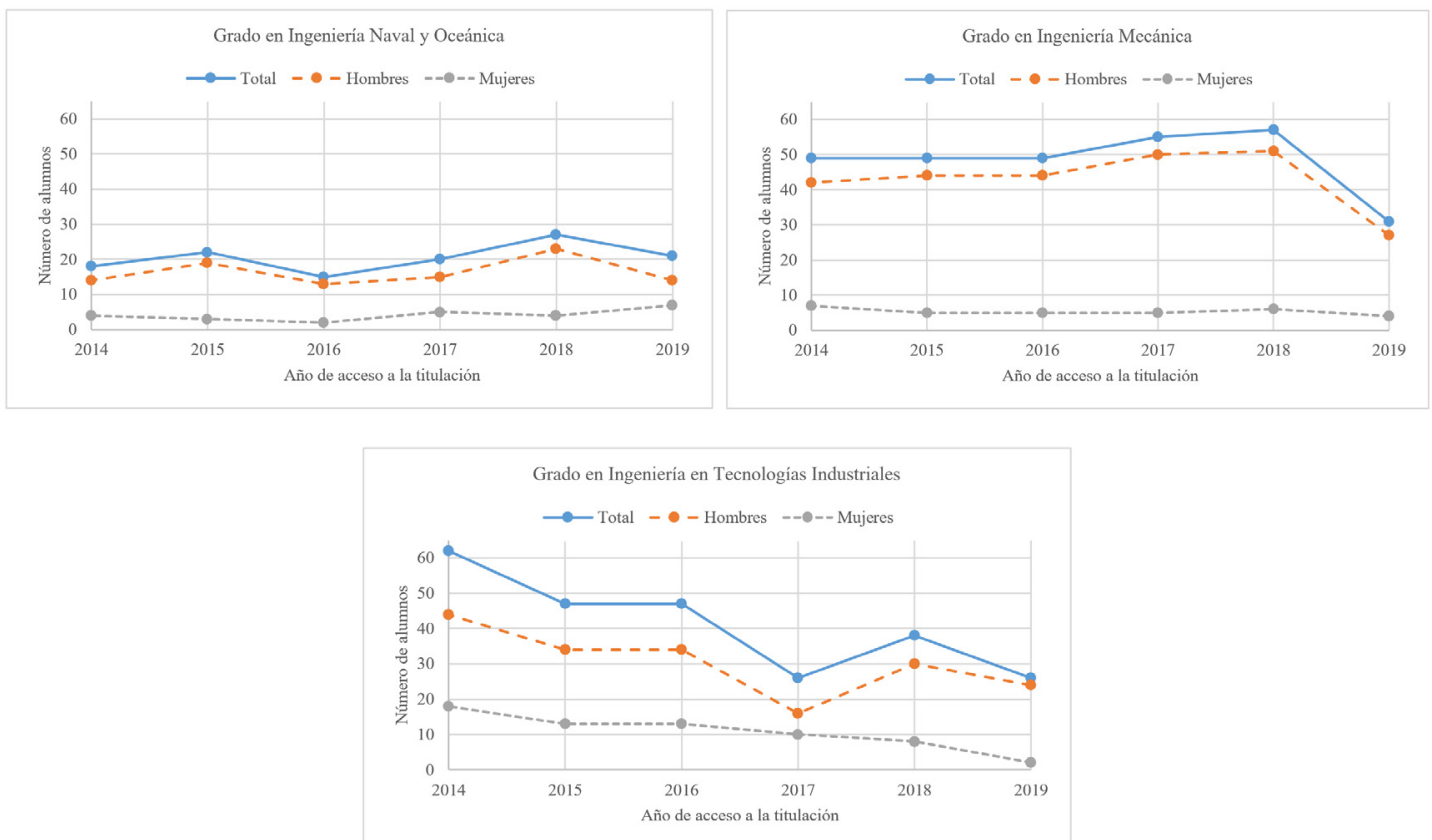

Figura 4. Número de alumnos de nuevo ingreso matriculados en las titulaciones de grado de la EPS. Fuente: Portal de Transparencia de la UDC ix / Subdirección de Calidad de la EPS.

Aunque estos hechos pudiesen hacer sugerir que la efectividad de las actividades llevadas a cabo ha sido muy limitada, esto puede en parte ponerse en duda si al mismo tiempo se observan los resultados mostrados en la Figura 5. Estos han sido obtenidos de una serie de encuestas realizadas a los alumnos de nueva matrícula en la Escola Politécnica Superior durante la "Semana de Acogida" del curso 2019/2020 (que se celebra la primera semana de curso), donde 
puede apreciarse que casi un $40 \%$ de los alumnos de nueva matrícula encuestados habían asistido a alguna de las actividades de difusión que se habían realizado, siendo las de mayor preponderancia las organizadas por el SAPE o concertadas por los propios centros educativos a título individual, lo que sugiere un elevado alcance de las actividades planteadas en el alumnado de nueva matrícula. Es por ello, que aunque se ha observado una disminución de la matrícula en el año 2019 (no así en 2018), las actividades realizadas han tenido repercusión y, probablemente, puedan haber minorado esta disminución de alumnado.

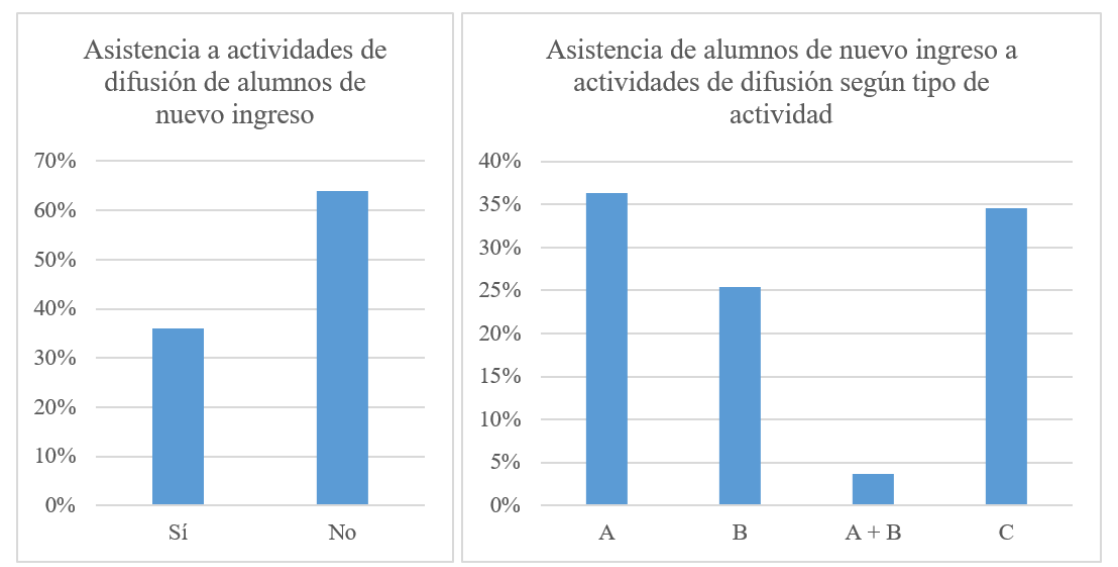

Figura 5. Asistencia previa a actividades de difusión/divulgación de titulaciones de los alumnos de nuevo ingreso en la Escola Politécnica Superior. Fuente: Subdirección de Calidad de la EPS.

A - SAPE/Visitas concertadas por centros educativos; B - Día de la Ingeniería; C - Otras.

\section{CONCLUSIONES}

En este trabajo se han expuesto las actividades de enseñanza realizadas por el Grupo de Innovación Educativa de la UDC CITEX como nuevo instrumento metodológico, formado por docentes de las titulaciones relacionadas con la Ingeniería Naval y Oceánica y la Ingeniería Industrial, y que tenían como objetivo mejorar los resultados académicos de las materias que imparten, así como la visibilidad y el conocimiento de dichas titulaciones por futuros alumnos, mediante el uso de actividades prácticas realizadas en una serie de instalaciones singulares de investigación situadas en el Centro de Investigaciones Tecnológicas (CIT) de la UDC en Ferrol. 
En ambos casos, las actividades realizadas giraban alrededor de la realización de ensayos experimentales y del uso de las instalaciones por parte de los alumnos para mejorar en la adquisición y comprensión de conceptos complejos o "threshold" en el caso de las actividades docentes, y para dar a conocer las titulaciones, las instalaciones de investigación y los resultados de la misma en el caso de las actividades de difusión y divulgación.

Respecto a los resultados obtenidos, en el caso de las actividades de enseñanza, los datos disponibles no permiten concluir de modo objetivo si las mismas contribuyen a una mejora en el aprendizaje del alumnado. Se hace indispensable el desarrollo de una metodología de evaluación y de consulta que permita obtener estos resultados.

En lo que respecta a las actividades de divulgación y difusión, los datos disponibles muestran incrementos en los alumnos de nueva matrícula de las titulaciones objetivo en el año 2018 y un descenso moderado en 2019, así como una alta asistencia a estas actividades por los alumnos de nueva matrícula. Aunque bajo estas condiciones son datos positivos, será necesario mejorar las encuestas a los alumnos de nuevo ingreso para valor en qué medida la asistencia a dichas actividades pudo influir en su decisión a la hora de elegir una titulación.

Finalmente, y en lo que se refiere a la divulgación de las propias instalaciones de investigación en el tejido social circundante a las mismas, las actividades realizadas recibieron una alta atención por los medios de comunicación por lo que, en este caso, los resultados tienen que clasificarse como satisfactorios.

\section{REFERENCIAS}

Biran, A. B., López-Pulido, R. 2014. Ship Hydrostatics and Stability. Waltham, MA: Elsevier. Blanco López, A. (2004). Relaciones entre la educación científica y la divulgación de la ciencia. Revista Eureka sobre enseñanza y divulgación de las ciencias, Volumen (1), Número (2), pp. 70-86.

Caamaño, A. (2012). ¿Cómo introducir la indagación en el aula? Alambique, Volumen (70), pp. 83-91. 
Conference of Ministers responsible for Higher Education. 2005. The European Higher Education Area - Achieving the Goals. Communiqué of the Conference of Ministers responsible for Higher Education. Bergen.

Delors, J. 1996. La educación encierra un tesoro. Madrid: Santillana/UNESCO.

European University Association. 2003. Graz Declaration 2003. Forward from Berlin: the Role of the Universities. Bruselas.

Knight, D.B., Callaghan, D.P., Baldock, T.E. \& Meyer, J.H.F. (2014). Identifying threshold concepts: case study of an open catchment hydraulics course. European Journal of Engineering Education, Volumen (39), Número (2), pp. 125-142.

Land, R., Meyer, J. H., \& Flanagan, M. T. (Eds.). (2016). Threshold concepts in practice. Springer.

MECD (2016). Datos y cifras del Sistema Universitario español. Curso 2015/2016. Secretaría General Técnica.

Míguez González, M., Bulian, G. (2018). Influence of ship dynamics modelling on the prediction of fishing vessels roll response in beam and longitudinal waves. Ocean Engineering, Volumen (148), pp. 312-330.

OECD. (2008). Encouraging Student Interest in Science and Technology Studies. Paris: OECD Publishing.

Osborne, J., Simon, S., \& Collins, S. (2003). Attitudes towards science: A review of the literature and its implications. International Journal of Science Education, Volumen (25), Número (9), pp. 1049-1079.

Universidade da Coruña. 2013. Plan estratéxico UDC 2020. Coruña: UDC.

\footnotetext{
'www.udc.es/avaliemos/

ii eps.udc.es

iii www.udc.es/campusindustrial

iv www.udc.es/sape

${ }^{v}$ cit.udc.es

vi www.firstlegoleague.es

vii https://www.lavozdegalicia.es/noticia/ferrol/ferrol/2019/01/19/oda-ingenerias-campus/0003_201901F19C3992.htm

viii $h$ ttps://www.youtube.com/watch?v=nRKBWG5r-hU

${ }^{\text {ix }}$ www.udc.es/transparencia/
} 
